\title{
GANHOS EM SAÚDE FAMILIAR SENSÍVEIS AO MODELO DINÂMICO DE AVALIAÇÃO/INTERVENÇÃO FAMILIAR
}

\author{
Family health gains sensitive to the Dynamic Family Assessment/Intervention Model
}

Beneficios en la salud familiar sensibles al Modelo Dinámico de Evaluación/Intervención Familiar

\author{
Manuela Ferreira, ${ }^{*}$ Catarina Pereira, ${ }^{* *}$ Maria João Rodrigues, ${ }^{* *}$ Mariana Paiva,,${ }^{* *}$ Vânia Arrojado, ${ }^{* *}$ Maria \\ Henriqueta Figueiredo ${ }^{* * *}$
}

\section{RESUMO}

Enquadramento: o Modelo Dinâmico de Avaliação e Intervenção Familiar (MDAIF) é um referencial teórico e operativo que pretende dar resposta às necessidades dos enfermeiros no cuidado às famílias. Objetivo: avaliar os ganhos em saúde sensíveis aos cuidados de enfermagem prestados por estudantes colaborativamente com as famílias segundo o MDAIF. Metodologia: trata-se de um estudo descritivo, transversal, de natureza quantitativa, com uma amostra de conveniência, constituída por 35 famílias. A colheita de dados foi realizada a partir dos registos de enfermagem dos cuidados prestados às famílias, sujeitos posteriormente a análise descritiva. Resultados: o MDAIF permitiu aos estudantes obter em ganhos em saúde junto das famílias. Dos diagnósticos formulados destacam-se pela prevalência os da dimensão do desenvolvimento, seguidos dos da dimensão funcional e finalmente os da dimensão estrutural. Registaram-se ganhos em saúde sensíveis aos cuidados de enfermagem em todas as áreas de atenção das dimensões, com uma modificação positiva no estado dos diagnósticos de enfermagem de $80,8 \%$ do antes para após a intervenção. Conclusão: os estudantes de enfermagem, fundamentados no MDAIF, avaliaram e interviram colaborativamente com as famílias, obtendo ganhos em saúde familiar. Recomenda-se a disseminação generalizada do MDAIF no cuidado às famílias.

Palavras-chave: diagnóstico de enfermagem; enfermagem familiar; enfermeiras de saúde da família; saúde da família

*PhD, Professor adjunto na Escola Superior de Saúde Norte da Cruz Vermelha Portuguesa/CINTESIS

https://orcid.org/0000-0003-0019-9534 autor de correspondência: manuela.ferreira@essnortecvp.pt

${ }^{* *}$ RN, Enfermeira, Escola Superior de Saúde Norte da Cruz Vermelha Portuguesa

${ }^{* * *} \mathrm{PhD}$, Professor coordenador na Escola Superior de Enfermagem do Porto/CINTESIS https://orcid.org/0000-0001-7902-9751

Como referenciar:

Ferreira, M., Pereira, C., Rodrigues, M.J., Paiva, M., \& Figueiredo M.H. (2020). Ganhos em saúde familiar sensíveis ao modelo dinâmico de avaliação/intervenção familiar. Revista de Investigação \& Inovação em Saúde, 3(2), 7-20. doi.org/10.37914/ riis.v3i2.84

Recebido para publicação em: 05/07/2020 Aceite para publicação: 24/11/2020

\section{ABSTRACT}

Background: the Dynamic Model of Family Assessment and Intervention (MDAIF) is a theoretical and operational framework that aims to respond to the needs of nurses in caring for families. Objective: to evaluate health gains sensitive to nursing care provided by students collaboratively with families according to the MDAIF. Methodology: this is a descriptive, cross-sectional, quantitative study, with a convenience sample, consisting of 35 families. Data collection was performed from the nursing records of caring provided to families, which were subsequently subjected to a descriptive analysis. Results: the MDAIF allowed students to obtain health gains beside their families. From the diagnoses formulated, those of the development dimension stand out, followed by those of the functional dimension and finally by those of the structural dimension. There were gains in health that were sensitive to nursing care in all areas of care of the dimensions, with a positive change in the state of nursing diagnoses from $80.8 \%$ from before to after the intervention. Conclusion: nursing students, based on the MDAIF, evaluated and intervened collaboratively with families, obtaining gains in family health. Widespread dissemination of MDAIF in family care is recommended.

Keywords: nursing diagnosis; family nursing; family nurse practitioners; family health

\section{RESUMEN}

Marco Contextual: el Modelo Dinámico de Evaluación e Intervención Familiar (MDAIF) es un marco teórico y operativo que tiene como objetivo dar respuesta a las necesidades del enfermero en el cuidado de la familia. Objetivo: evaluar las mejoras en salud sensibles a los cuidados de enfermería proporcionadods por los estudiantes en colaboración con las familias según el MDAIF. Metodología: se trata de un estudio descriptivo, transversal, cuantitativo, con muestra de conveniencia, conformada por 35 familias. La recolección de datos se realizó a partir de los registros de enfermería de cuidados proporcionados a las familias, los cuales fueron posteriormente sometidos a un análisis descriptivo. Resultados: el MDAIF permitió a los estudiantes obtener beneficios para la salud de sus familias. De los diagnósticos formulados destacan los de la dimensión de desarrollo, seguidos de los de la dimensión funcional y con menor prevalencia los de la dimensión estructural. Hubo avances en salud sensibles al cuidado de enfermería en todas las áreas de cuidado de las dimensiones, con un cambio positivo en el estado de los diagnósticos de enfermería del $80,8 \%$ antes al después de la intervención. Conclusión: los estudiantes de enfermería, con base en el MDAIF, evaluaron e intervinieron de manera colaborativa con las familias, obteniendo mejoras en salud familiar. Se recomienda una amplia difusión del MDAIF en la atención familiar.

Palavras clave: diagnóstico de enfermería; enfermería de la familia; enfermeiras de família; salud de la família 
Ganhos em saúde familiar sensíveis ao modelo dinâmico de avaliação/intervenção familiar

\section{INTRODUÇÃO}

A enfermagem de saúde familiar tem como foco os cuidados de enfermagem à família como um todo, sem esquecer os seus membros individualmente (Shivalli, Majra, Akshaya, \& Qadiri, 2015; Figueiredo, 2012). Os cuidados de enfermagem à família desenvolvem-se numa relação terapêutica, dinâmica e recursiva caraterizada pela elaboração de soluções promotoras de mudança, a partir das potencialidades da família e respeitando a sua realidade. 0 enfermeiro apresenta um papel de observadorparticipante neste processo evolutivo de mudança e facilitador de construção de soluções de forma colaborativa com a família (Kokorelias, Gignac, Naglie, \& Cameron, 2019; Figueiredo, 2012). O MDAIF foi adotado pela Ordem dos Enfermeiros para dar respostas às necessidades dos enfermeiros portugueses (Figueiredo, 2012) e é atualmente adotado por muitas Escolas Superiores de Enfermagem/Saúde no ensino de enfermagem e pelos enfermeiros nas Unidades de Saúde Familiar e Unidades de Cuidados de Saúde Personalizados dos Cuidados de Saúde Primários em Portugal.

Constituiu objetivo geral deste estudo avaliar os ganhos em saúde sensíveis aos cuidados de enfermagem prestados por estudantes da licenciatura colaborativamente com as famílias segundo o MDAIF. Tem ainda como objetivos específicos: caracterizar as famílias estudadas; analisar as áreas de atenção, focos, diagnósticos de enfermagem e resultados de saúde sensíveis aos cuidados de enfermagem, a partir das modificações positivas no estado dos diagnósticos de enfermagem (Ordem Enfermeiros, 2007), definidos à priori no MDAIF (Figueiredo, 2012).

\section{ENQUADRAMENTO/ FUNDAMENTAÇÃO TEÓRICA}

A família é considerada como um grupo de pessoas que convivem no mesmo lar e estão ligadas por vínculos afetivos possuindo a sua identidade $\mathrm{e}$ unicidade (Russell, Coleman, \& Ganong, 2018; Figueiredo, 2012). Família é o todo e partes ao mesmo tempo, e apresenta caraterísticas que são mais e menos do que a soma das partes sendo impossível fazer a sua descrição apenas com base nos indivíduos que a constituem, o que significa que cada um tem uma estrutura relacional e complexa possuindo diferentes funções e papeis, bem como, expetativas sociais, não havendo assim duas famílias iguais. As alterações na família, decorrentes do ciclo vital, afetam quer as partes, quer o todo, em que ambos tendem para a solução e mudança necessária para o equilíbrio dinâmico da unidade familiar. Os seus elementos (subsistemas familiares) desenvolvem determinadas funções que vão sendo transformadas ao longo do ciclo vital (Figueiredo, 2012). O tipo de família permite diferenciar a sua constituição e os vínculos entre os indivíduos, podendo condicionar a avaliação e a identificação de focos sensíveis aos cuidados de enfermagem. Tal como acontece no indivíduo, a família também apresenta um ciclo vital, que corresponde às mudanças e transformações da família ao longo do seu percurso evolutivo (Figueiredo, 2012), que condicionam a avaliação e a identificação de focos sensíveis aos cuidados de enfermagem.

A Saúde Familiar é um estado subjetivo caraterizado pelo conjunto de particularidades associadas à maximização do potencial de saúde da família. A saúde familiar depende da saúde e da doença de cada individuo que afeta a funcionalidade da família, por 


\section{Ganhos em saúde familiar sensíveis ao modelo dinâmico de avaliação/intervenção familiar}

outro lado a funcionalidade da família afeta a saúde de cada individuo que a constitui (Kholifah, Nursalam, Adriani, Ahsan, \& Susanto, 2016; Shivalli et al., 2015; Figueiredo, 2012). A saúde familiar traduz-se na capacidade da família para criar estratégias que permita a sua funcionalidade, mantendo a organização, produzindo mudanças estruturais e dar respostas às necessidades individuais dos seus membros. Na família, cada individuo desenvolve e promove o conceito de saúde em que adquire hábitos saudáveis e possui um papel de mediador ou de impacte que ajuda na recuperação da saúde quando um dos membros da família necessita de cuidados (Figueiredo, 2012).

O papel do enfermeiro de família em Portugal existe integrado numa equipa multidisciplinar com a responsabilidade de prestar cuidados de enfermagem gerais à família em todas as suas fases do seu ciclo vital, contribuindo também para a ligação entre os membros da família. Os enfermeiros, na sua atuação, devem ter em atenção: a experiência que a família tem sobre si própria, o seu passado, presente, e perspetivas futuras, a doença, descrevendo o significado e o impacte que a doença tem, não só na pessoa, mas também em toda a sua família; os cuidados que devem ser prestados à pessoa pela sua família ou prestados à família como cliente; intervenções de enfermagem que tenham como foco as estratégias para trabalhar a família nas suas situações de saúde-doença (Kokorelias et al., 2019; Figueiredo, 2012).

Os cuidados de enfermagem centram-se na interação entre o enfermeiro e a família, estabelecendo um processo interpessoal, significativo e terapêutico (Waldow, 2014; Silva, Costa, \& Silva, 2013) e têm como objetivo promover a participação dos seus membros em todas as etapas, promovendo a mudança na família, a utilização de estratégias de coping, que mobilize as suas forças e dos recursos disponíveis para ajudar a família a identificar e solucionar autonomamente os seus problemas face aos seus projetos de vida/saúde (Waldow, 2014; Figueiredo, 2012). Os focos de enfermagem em saúde familiar são a promoção de projetos de saúde da família e as intervenções correspondem a elementos promotores de capacitação da família (Silva et al., 2013), dando assim respostas afetivas, cognitivas e comportamentais ao sistema terapêutico (Figueiredo, 2012). Desta forma, as intervenções de enfermagem centram-se na família e em cada individuo que a constitui.

Os principais componentes dos modelos teóricos para facilitar o atendimento centrado na família incluem: 1) colaboração entre familiares e profissionais de saúde, 2) consideração dos contextos familiares, 3) políticas e procedimentos e 4) educação profissional do utente, da família e da saúde (Kokorelias et al., 2019). O MDAIF é considerado um referencial teórico e operativo, que pretende dar as respostas as necessidades dos enfermeiros, responsáveis pelos cuidados de saúde familiar e desenvolvimento de práticas de enfermagem relacionados com a família (Figueiredo, 2012). Este modelo baseia-se no pensamento sistémico, em que a família é o principal foco de atenção, sem esquecer os membros da família individualmente (Silva et al., 2013).

O MDAIF, com os seus princípios e postulados, centra-se nas áreas de atenção familiar por domínios avaliativos, onde especifica três grandes dimensões 
de avaliação e intervenção familiar: estrutural, de desenvolvimento e funcional (Figueiredo, 2012). A dimensão estrutural, consiste na avaliação e intervenção na estrutura interna, externa e de contexto, com uma visão sobre a composição da família, o vínculo entre os seus membros e entre estes e outros elementos significativos como a família alargada, assim como no contexto onde a família se insere (Russell et al., 2018; Seibel et al., 2017). A dimensão desenvolvimento simplifica a compreensão dos fenómenos da família, associados à sua trajetória evolutiva a nível funcional, estrutural e inter-relacional. A avaliação e intervenção que determina a forma de como os membros da família interagem uns com os outros e que se centra nas atividades do quotidiano da vida da família está presente na dimensão funcional. A avaliação e intervenção colaborativa da família é fundamental para identificar as necessidades da família, realçar as forças e o potencial da família para a evolução através da ligação dos membros da família com os enfermeiros de saúde familiar (Tavakol, Nasrabadi, Moghadam, Salehiniya, \& Rezaei, 2017; Figueiredo, 2012). O MDAIF baseia-se no processo de enfermagem com a finalidade capacitar a família para a possível mudança do seu funcionamento cognitivo, afetivo e comportamental numa evolução contínua, entre estadios de menor complexidade para estadios de maior complexidade, enquadradores do seu ciclo vital, traduzindo ganhos em saúde familiar sensíveis aos cuidados de enfermagem (Figueiredo, 2012).

Os modelos teóricos de avaliação e intervenção na família facilitam fundamento conceptual e operativo compreensível, são fáceis de aplicar e habilitam os enfermeiros de família de competências para ajudar as famílias (Kholifah et al., 2016; Leahey \& Wright, 2016), mas cuja eficácia carece de maior evidencia científica (Kokorelias et al., 2019), facto que justifica a realização do presente estudo.

\section{METODOLOGIA}

Estudo descritivo, transversal, de natureza quantitativa, cuja amostra é de conveniência, constituída por 35 processos clínicos de famílias avaliadas e intervencionadas segundo o MDAIF pelos estudantes do Curso de Licenciatura em Enfermagem, no âmbito do ensino clínico da área de enfermagem de saúde familiar de uma escola superior de saúde da Região Centro de Portugal, nos anos letivos 2018/2019 e 2019/2020. Foram incluídos neste estudo todos os processos das famílias estudadas, tendo sido excluídos os processos familiares em cujas famílias não tenham dado consentimento informado livre e esclarecido escrito para a aplicação do estudo de caso à família, ou sem consentimento informado por parte dos estudantes, para a utilização dos estudos de caso ou cuja abordagem feita às famílias pelos estudantes não siga a matriz operativa do MDAIF.

O instrumento de colheita de dados utilizado foi uma grelha de registos definida à priori a partir do MDAIF, constituído por quatro partes. A primeira parte contempla dados de caracterização das famílias (tipo de família, subsistemas familiares, tipo e intensidade de contacto dos elementos da família e, função das relações com a família extensa, sistemas mais amplos (comunidade escola, colegas de trabalho, profissionais de saúde, colaboradores das instituições que apoiem a família), classe social, etapa do ciclo 


\section{Ganhos em saúde familiar sensíveis ao modelo dinâmico de avaliação/intervenção familiar}

vital da família, e família com membro dependente). A segunda parte integra os diagnósticos de enfermagem segundo as três dimensões do MDAIF. As intervenções de enfermagem fazem parte da terceira parte do questionário. Na última parte do instrumento de colheita de dados apresentam-se os resultados de enfermagem.

A recolha de dados a partir do processo clínico das famílias foi feita por dois investigadores, de forma independente, com o propósito de aumentar a homogeneidade e reduzir desvios na categorização dos dados. Sempre que se verificaram desvios à análise dos dados, pelos investigadores, foi feita uma reanálise para permitir o consenso. Para a análise dos dados foram utilizadas medidas estatísticas descritivas, com recurso ao software Statistical Package for the Social Sciences ${ }^{\circledR}$, versão 21 . Este estudo teve aprovação pela Comissão de Ética (Parecer № 08/2019) e Unidade de Investigação e Desenvolvimento da Escola Superior de Saúde (2019015), tendo sido cumpridos os princípios éticos previstos.

\section{RESULTADOS}

As famílias estudadas são maioritariamente nucleares ( $n=21 ; 61,8 \%)$, seguidas das famílias alargadas $\quad(n=5 ; \quad 14,7 \%) . \quad$ Com menos representatividade verifica-se, por ordem decrescente, as famílias casal ( $n=3 ; 8,8 \%$ ), as famílias reconstruídas ( $n=2 ; 5,9 \%)$ e com igual representatividade a família monoparental liderada pela mulher ( $n=1 ; 2,9 \%)$, família de coabitação $(n=1$; $2,9 \%$ ) e família unipessoal ( $n=1 ; 2,9 \%)$. Não foi possível determinar a tipologia de uma das famílias, pela ausência de registo.
Relativamente à classe social das famílias estudadas a maioria enquadra-se na classe média ( $n=15 ; 42,9 \%)$, seguida da classe média baixa $(n=8 ; 22,9 \%)$, da classe média alta $(n=7 ; 20 \%)$, da classe baixa $(n=4 ; 11,4 \%)$ e finalmente, com menos representatividade a classe alta ( $n=1 ; 2,9 \%)$. Em relação à etapa do ciclo vital familiar, dos 31 registos, mais de metade da amostra são famílias com filhos pequenos ( $n=17 ; 54,8 \%$ ) e cerca de $32,3 \%$ ( $n=10$ ) são famílias com filhos adultos, sendo a minoria famílias com filhos na escola $(n=3$; $9,7 \%$ ) e famílias com filhos adolescentes ( $n=1 ; 2,9 \%$ ). A maioria das famílias com registo quanto à existência de subsistema conjugal $(n=31)$ apresenta subsistema conjugal ( $n=21 ; 67,7 \%$ ) e destes, 32,3\% dos casais $(n=10)$ estão em idade fértil. Das famílias estudadas $42,9 \%$ apresentam subsistema parental $(n=15)$.Relativamente ao tipo de contacto da família extensa, dos 34 registos, o mais usual é o contacto presencial $(94,1 \% ; n=32)$ e o telefónico $(82,4 \% ; n=28)$, a intensidade/frequência de contacto mais apresentada foi a diária $(67,6 \% ; n=23)$ e semanal $(41,2 \% ; n=14)$. Dos 34 registos quanto às funções da rede da família extensa, apresentam-se por ordem decrescente de prevalência: o apoio emocional (91,2\%; $n=31)$; a companhia social $(73,5 \% ; n=25)$; o guia cognitivo e de conselhos (32,4\%; $n=11)$; e ainda a ajuda material e de serviços $(29,4 \% ; n=10)$.

Os sistemas mais amplos apresentados nas 35 famílias em estudo são por ordem decrescente: os serviços de saúde (71,4\%; $n=25)$; amigos (65,7\%; $n=23)$; colegas de trabalho $(60,0 \% ; n=21)$; grupos de lazer e cultura (40,0\%; $n=14)$; vizinhos $(28,6 \% ; n=10)$; comunidade escolar (28,6\%; $n=10)$; e grupos religiosos $(25,7 \%$; $n=9)$. 
Das famílias estudadas e com registo quanto à existência de membros dependentes $(n=22)$ apenas $27,3 \%(n=6)$ apresenta membros dependentes. No que se refere aos níveis de dependência, dos seis registos com a variável nível de dependência presente, a sua maioria $(83,3 \% ; n=5)$, apresenta dependência ao nível do autocuidado de higiene, vestuário, atividade de lazer, atividade física, gestão do regime terapêutico, autovigilância e medicação, seguida da dependência no autocuidado de ir ao sanitário e sono-repouso $(66,6 \% ; n=4)$ e finalmente, a dependência no autocuidado de comer e beber (50,0\%; $n=3)$. Todas as famílias com membros dependentes $(n=6)$ apresentaram como prestador de cuidados principal um membro da família, este é apoiado em 50,0\% destas famílias por um cuidador formal/ajudante familiar $(n=3)$.
Da análise da Tabela 1 podemos concluir que, na avaliação inicial, antes da intervenção dos estudantes de enfermagem (intervenção colaborativa com as famílias), verifica-se um conjunto diversificado de diagnósticos de enfermagem à família (219), distribuídos pelas diferentes áreas de atenção das dimensões do MDAIF sendo que, em média, existem 6,3 $\pm 3,8$ diagnósticos de enfermagem por família. De todas as dimensões a que apresenta uma menor frequência de diagnósticos de enfermagem é a dimensão estrutural, com 35 diagnósticos $(16,0 \%)$, seguida da dimensão funcional com 63 (28,8\%) diagnósticos de enfermagem e por último, a mais representativa, a dimensão desenvolvimento, com um total de 121 (55,2\%) diagnósticos de enfermagem.

\section{Tabela 1}

Evolução do número de diagnósticos de Enfermagem que requerem intervenções de enfermagem segundo as dimensões e áreas de atenção antes e após a intervenção de enfermagem

\begin{tabular}{|c|c|c|c|c|}
\hline Dimensão & Áreas de atenção & Diagnósticos de Enfermagem & Antes (n) & Após (n) \\
\hline \multirow[b]{6}{*}{ Estrutural } & Rendimento Familiar & Rendimento Familiar Insuficiente & 6 & 4 \\
\hline & \multirow[t]{2}{*}{ Edifício residencial } & Edifício Residencial Não Seguro & 5 & 1 \\
\hline & & Edifício Residencial Negligenciado & 4 & 0 \\
\hline & Precaução de Segurança & Precaução de Segurança Não Demonstrada & 5 & 1 \\
\hline & Abastecimento de água & Abastecimento de Água Não Adequado & 7 & 2 \\
\hline & Animal doméstico & Animal Doméstico Negligenciado & 8 & 1 \\
\hline & \multirow{5}{*}{ Satisfação conjugal } & Satisfação Conjugal Não Mantida & 19 & 3 \\
\hline & & Relação Dinâmica Disfuncional & 17 & 2 \\
\hline & & Comunicação Não eficaz & 5 & 0 \\
\hline & & Interação Sexual Não Adequada & 1 & 0 \\
\hline & & Função Sexual Comprometida & 2 & 0 \\
\hline & \multirow[t]{5}{*}{ Planeamento familiar } & Planeamento Familiar Ineficaz & 7 & 3 \\
\hline & & Fertilidade Comprometida & 1 & 1 \\
\hline & & $\begin{array}{l}\text { Conhecimento Sobre Vigilância Pré-concecional } \\
\text { não demonstrado }\end{array}$ & 2 & 1 \\
\hline & & Uso de Contracetivo Não adequado & 4 & 1 \\
\hline & & $\begin{array}{llll}\begin{array}{l}\text { Conhecimento sobre } \\
\text { demonstrado }\end{array} & \text { Reprodução não } \\
\end{array}$ & 4 & 1 \\
\hline & \multirow[t]{2}{*}{ Adaptação à gravidez } & Adaptação à Gravidez Não Adequada & 1 & 0 \\
\hline Desenvolvimental & & Conhecimento não demonstrado & 1 & 0 \\
\hline
\end{tabular}


Ganhos em saúde familiar sensíveis ao modelo dinâmico de avaliação/intervenção familiar

\begin{tabular}{|c|c|c|c|c|}
\hline & & Comunicação Não eficaz & 0 & 0 \\
\hline & & Comportamento de Adesão não demonstrado & 1 & 0 \\
\hline & Papel parental & Papel parental não adequado & 23 & 3 \\
\hline & & Conhecimento do papel não demonstrado & 18 & 0 \\
\hline & & Comportamento de Adesão não demonstrado & 10 & 2 \\
\hline & & Consenso de papel: não & 2 & 0 \\
\hline & & Conflito de papel & 2 & 0 \\
\hline & & Saturação de papel & 3 & 1 \\
\hline \multirow[b]{11}{*}{ Funcional } & \multirow{6}{*}{$\begin{array}{l}\text { Papel de prestador de } \\
\text { cuidados }\end{array}$} & Papel de prestador de Cuidados Não Adequado & 5 & 0 \\
\hline & & Conhecimento de papel não demonstrado & 5 & 1 \\
\hline & & Comportamento de Adesão não demonstrado & 3 & 1 \\
\hline & & Consenso de papel: não & 0 & 0 \\
\hline & & Conflito de papel & 0 & 0 \\
\hline & & Saturação de papel & 2 & 0 \\
\hline & \multirow[t]{5}{*}{ Processo familiar } & Processo Familiar Disfuncional & 15 & 4 \\
\hline & & Comunicação familiar não eficaz & 13 & 3 \\
\hline & & Coping familiar ineficaz & 9 & 2 \\
\hline & & Interação de Papeis não eficaz & 6 & 3 \\
\hline & & Relação Dinâmica Disfuncional & 5 & 1 \\
\hline \multicolumn{3}{|l|}{ TOTAL } & 219 & 42 \\
\hline
\end{tabular}

Na avaliação final, após a intervenção adequada à família a partir do preconizado para cada diagnóstico pelo MDAIF, verifica-se uma modificação positiva nos estados de 177 diagnósticos de enfermagem. A dimensão desenvolvimento, com 18 diagnósticos, continua a ser a dimensão com maior proporção de diagnósticos de enfermagem (42,9\%), seguida da dimensão funcional, com 15 diagnósticos de enfermagem (35,7\%) e finalmente a dimensão estrutural, com 9 diagnósticos de enfermagem $(21,4 \%)$ (Figura 1$)$.

Verifica-se da avaliação inicial para a avaliação final, após a intervenção dos estudantes de enfermagem, uma modificação positiva nos estados dos diagnósticos de Enfermagem de $80,8 \%$, o que revela um impacte positivo na saúde familiar.

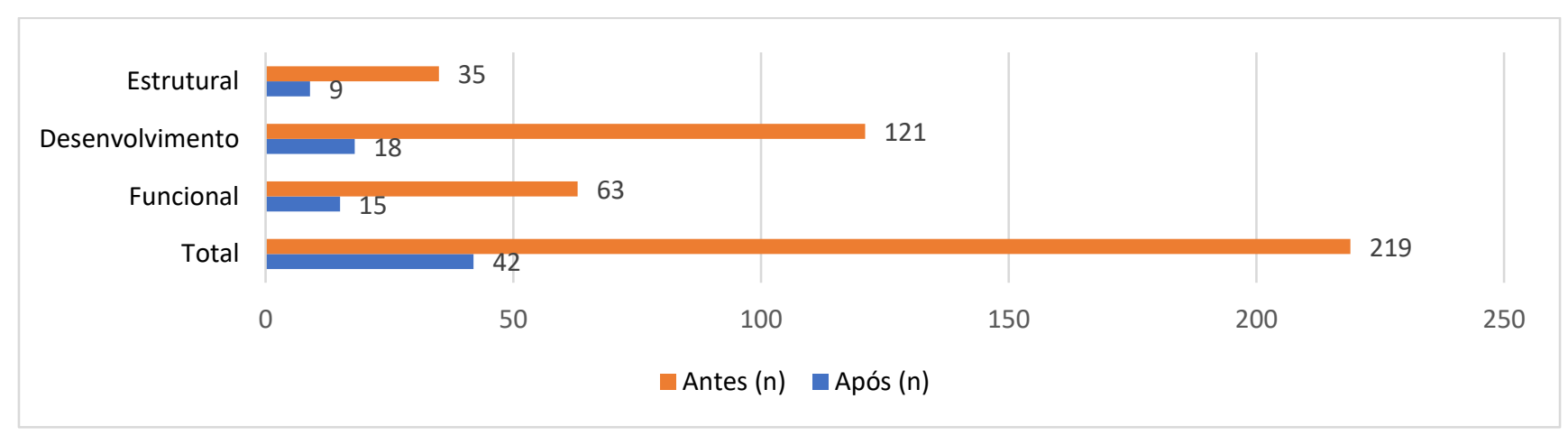

Figura 1

Gráfico da evolução do número de diagnósticos de enfermagem que careciam de intervenção antes e após da intervenção colaborativa com as famílias, segundo as dimensões do MDAIF 
Da análise da Figura 2, relativa à dimensão estrutural do MDAIF verifica-se que $22,9 \%$ das famílias, na avaliação inicial, apresentavam como diagnóstico animal doméstico negligenciado $(n=8), 20 \%$ das famílias apresentavam diagnóstico de abastecimento de água não adequado $(n=7), 19,1 \%$ das famílias apresentavam como diagnóstico de enfermagem o rendimento familiar insuficiente $(n=6)$. Após a aplicação dos cuidados de enfermagem colaborativos com a família, a partir das intervenções preconizados pelo MDAIF para cada diagnóstico, verifica-se a existência de ganhos em saúde sensíveis aos cuidados de enfermagem comprovados por evolução positiva dos estados dos diagnósticos em $100 \%$ das famílias com diagnóstico de edifício residencial negligenciado (4 família), 87,5\% das famílias com diagnóstico de animal doméstico negligenciado ( 7 famílias), $80 \%$ das famílias com diagnóstico de edifício residencial não seguro (4 famílias) e precaução de segurança não demonstrado (4 famílias), $71,4 \%$ das famílias com diagnóstico de abastecimento de água não adequado (5 famílias) e 33,3\% das famílias com diagnóstico de rendimento familiar insuficiente ( 2 famílias).

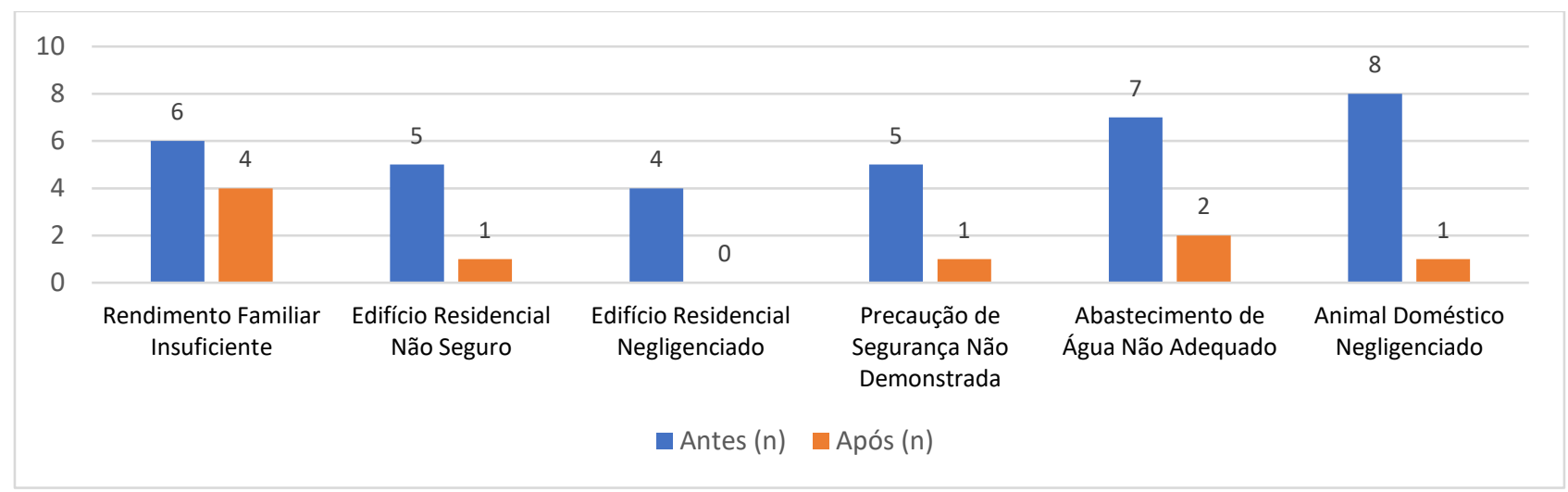

Figura 2

Gráfico da evolução do número de diagnósticos de enfermagem na dimensão estrutural do MDAIF que careciam de intervenção antes e após a intervenção de enfermagem colaborativa com as famílias

Na avaliação inicial da dimensão desenvolvimental das famílias, os diagnósticos de enfermagem que careciam de intervenção mais prevalentes foram o papel parental não adequado em $65,7 \% \quad(n=23)$, satisfação conjugal não mantida em 54,2\% ( $n=19)$, conhecimento do papel parental não demonstrado $51,4 \%$ ( $n=18)$, relação dinâmica disfuncional em 48,6\% ( $n=17)$, comportamento de adesão não demonstrado em $28,6 \%(n=10)$ e planeamento familiar ineficaz em $20 \%(n=7)$ (Figura 3$)$.
Após a aplicação dos cuidados de enfermagem colaborativos com a família, preconizados pelo MDAIF para cada diagnóstico de enfermagem e adaptados à família, verificaram-se ganhos em saúde sensíveis aos cuidados de enfermagem comprovados por uma redução em $100 \%$ das famílias com diagnóstico de satisfação conjugal, que carecem de cuidados de enfermagem: comunicação não eficaz (5 famílias), interação sexual não adequada (1 família) e função sexual não comprometida (2 famílias); adaptação à gravidez: não adequada (1 família), conhecimento e 
comportamento de adesão não demonstrado (1 família); papel parental: conhecimento de papel não demonstrado (18 famílias), ausência de consenso (2 famílias) e conflito de papel (2 famílias). 87,0\% das famílias viram resolvido, após a intervenção, o diagnóstico de papel parental não adequado (20 famílias), 84,2\% das famílias o diagnóstico de relação dinâmica disfuncional (15 famílias) e 80,0\% das famílias o diagnóstico de papel parental comportamento de adesão não demonstrado (8 famílias), 75,0\% das famílias o diagnóstico de uso de contracetivo não adequado (3 famílias) e conhecimento sobre reprodução não adequado (3 famílias), 66,6\% das famílias o diagnóstico de saturação de papel parental (2 famílias), 57,1\% das famílias o diagnóstico de planeamento familiar ineficaz (4 famílias) e finalmente $50 \%$ das famílias viram o diagnóstico de conhecimento de vigilância pré-concecional não demonstrado resolvido (1 família).

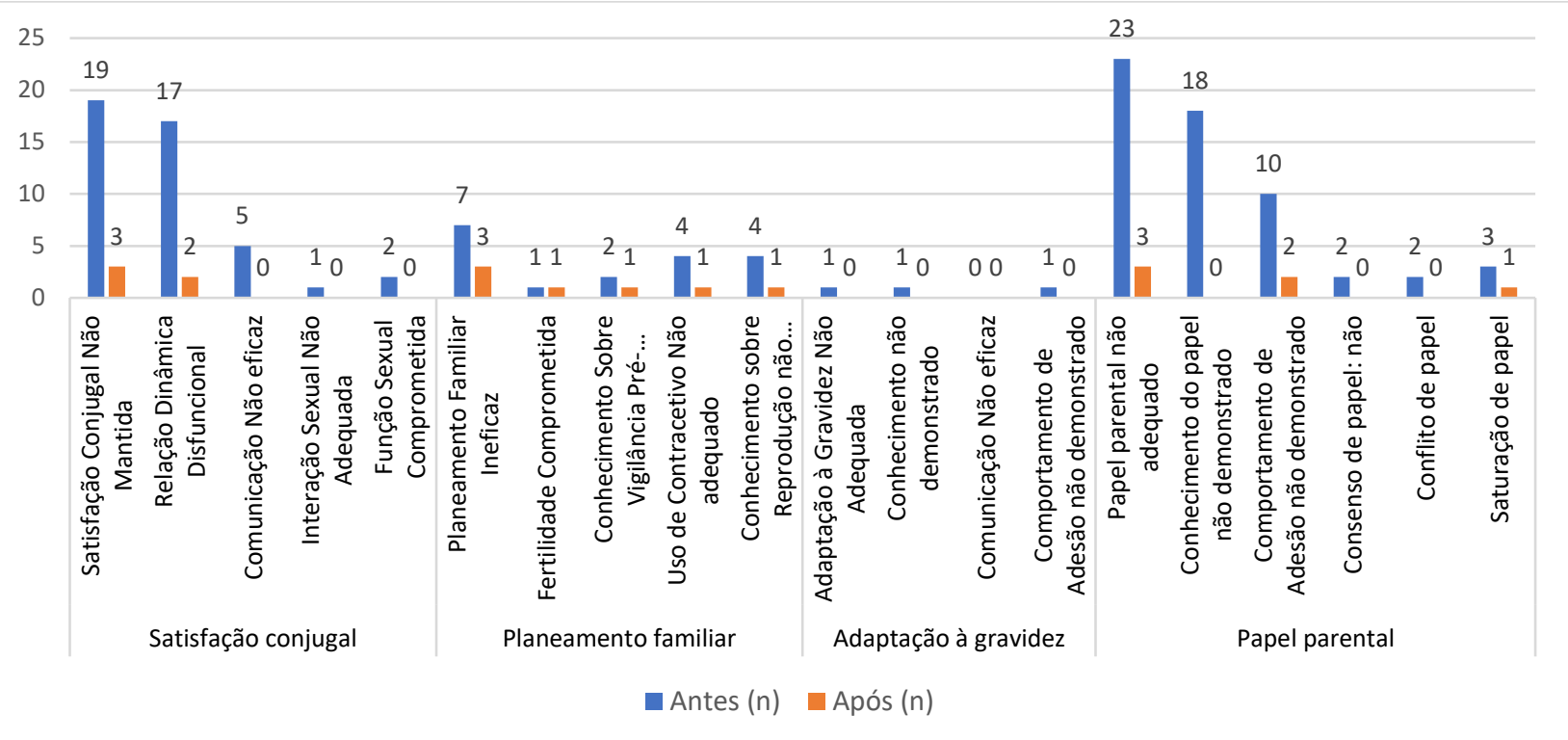

Figura 3

Gráfico da evolução do número de diagnósticos de enfermagem que careciam de intervenção nas áreas de atenção da dimensão desenvolvimental do MDAIF antes e após da intervenção de enfermagem colaborativa com as famílias

Após a aplicação dos cuidados de enfermagem colaborativos com a família na dimensão funcional, preconizados pelo MDAIF para cada diagnóstico de enfermagem e adaptados à família, verifica-se que 42,9\% das famílias, na avaliação inicial, apresentavam como diagnóstico papel processo familiar disfuncional ( $n=15), 37,1 \%$ das famílias apresentavam diagnóstico de comunicação familiar ineficaz $(n=13), 25,7 \%$ das famílias apresentavam como diagnóstico de enfermagem coping familiar ineficaz $(n=9), 17,1 \%$ das famílias apresentavam o diagnóstico de interação de papeis ineficaz $(n=6), \quad 14,3 \%$ das famílias apresentavam os diagnósticos de papel de prestador de cuidados não adequado $(n=5)$, conhecimento sobre 
papel de prestador de cuidados não adequado $(n=5)$, e relação dinâmica disfuncional $(n=5), 8,6 \%$ das famílias apresentavam o diagnóstico de comportamento de adesão ao papel parental não demonstrado $(n=3)$ e finalmente $5,7 \%$ das famílias apresentavam o diagnóstico de saturação do papel de prestador de cuidados (Figura 4).

Após a aplicação dos cuidados de enfermagem colaborativos com a família verificaram-se ganhos em saúde sensíveis aos cuidados de enfermagem, comprovados por uma redução em $100 \%$ das famílias com diagnóstico de papel de prestador de cuidados não adequado (5 famílias) e saturação do papel de prestador de cuidados (2 famílias). A maioria das famílias (80\%) viram resolvido o diagnóstico de enfermagem de conhecimento de papel de prestador de cuidados não demonstrado (4 famílias) e relação dinâmica disfuncional (4 famílias), 77,8\% das famílias resolveram o diagnóstico coping familiar ineficaz (7 famílias), 76,9\% das famílias o diagnóstico de comunicação familiar não eficaz (10 famílias), 73,3 das famílias o diagnóstico de processo familiar disfuncional (11 famílias), $66,6 \%$ das famílias o diagnóstico de conhecimento de adesão do papel de prestador de cuidados não demonstrado (2 famílias), e finalmente $50 \%$ das famílias resolveram o diagnóstico de interação de papeis não eficaz (3 famílias).

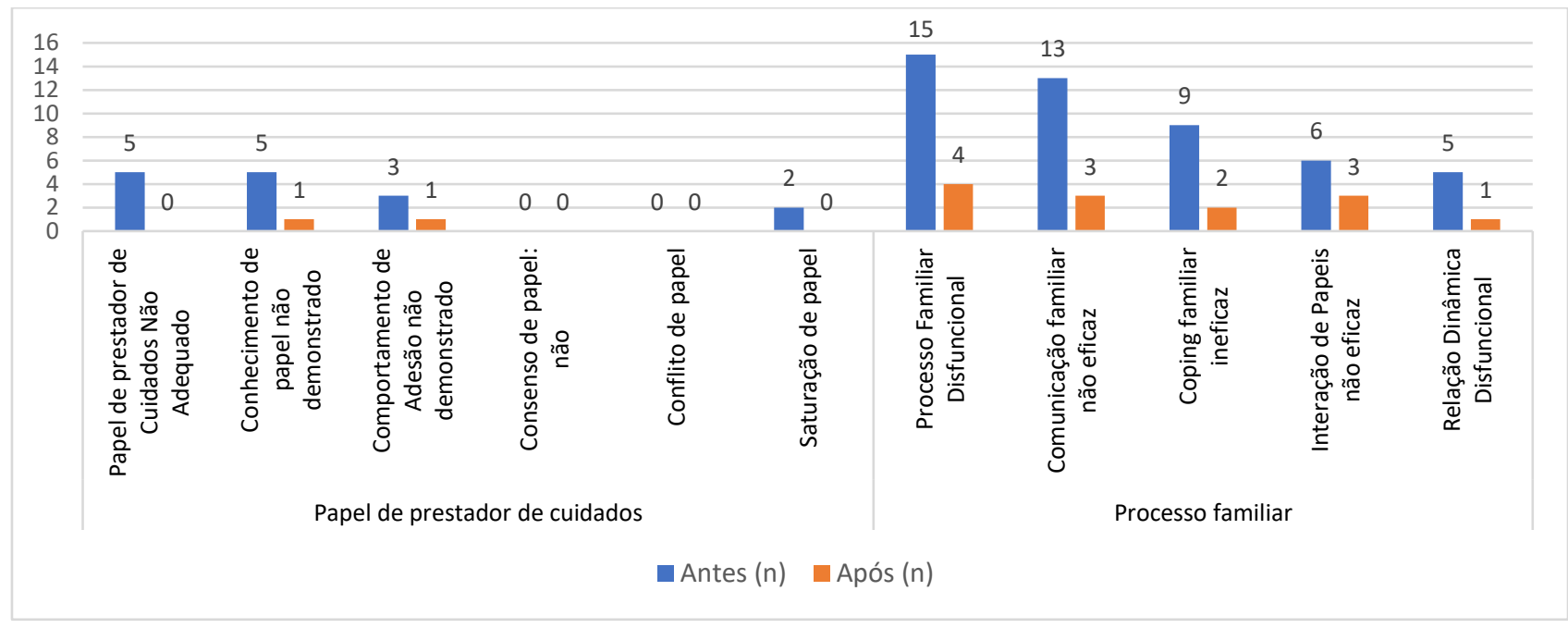

Figura 4

Gráfico da evolução do número de diagnósticos de enfermagem que carecem de intervenção nas áreas de atenção da dimensão funcional do MDAIF antes e após da intervenção de enfermagem colaborativa com as famílias

\section{DISCUSSÃO}

Maioritariamente as famílias estudadas são nucleares, proporção que se assemelha à proporção das famílias nucleares portuguesas registadas em 2011 (59\%) (Instituto Nacional de Estatística, 2013). Verifica-se um predomínio entre a classe média e média baixa (66\% das famílias), dado que revela alguma desigualdade social (Mauritti, Nunes \& Alves, 2019) 
entre riqueza e produção, evidenciando as novas tendências de concentração de riqueza no topo, com as classes médias a entrarem aos poucos num ciclo de frustração e crispação (Estanque, 2017).

Da avaliação familiar verifica-se uma grande proximidade das famílias com a sua rede de apoio social (frequência maioritariamente diária e sob a forma presencial ou por telefone) particularmente justificado pelo seu papel de apoio emocional, companhia social, guia cognitivo e de conselhos e ainda ajuda material e de serviços. Estes resultados revelam o papel protetor da rede de apoio sobre o funcionamento familiar e a qualidade das relações intrafamiliares, na sua estrutura, coesão e adaptabilidade (Seibel et al., 2017). Também Corrêa, Queiroz \& Fazito (2016) evidenciam o papel das redes como fonte de externalidades positiva (ou negativa) ao redistribuir recursos e reagir às políticas implementadas, influenciando e sendo influenciadas por elas. Os sistemas mais amplos apresentados nas famílias estudadas variam em função do estádio de desenvolvimento ciclo vital em que a família se encontra, da vivência por parte das famílias, de transições normativas e não normativas, das relações entre os subsistemas familiares (funcionamento familiar) e naturalmente, do contexto social no qual as famílias estão inseridas (Seibel et al., 2017), destacando-se, neste estudo, com maior expressão os serviços de saúde, amigos, colegas de trabalho/escola e vizinhos.

A aplicação do MDAIF junto de 35 famílias permitiu uma modificação positiva no estado de 177 diagnósticos de enfermagem que passaram de 219 para 42 diagnósticos, antes e após a intervenção, respetivamente. A dimensão desenvolvimento foi a que apresentou mais diagnósticos, justificado pela necessidade das famílias em de se adaptarem às transições normativas, seguindo-se a dimensão funcional, pela necessidade de ajuste ao desempenho de diferentes papeis na família e às questões da coesão, adaptabilidade e melhor funcionamento familiar e finalmente, a dimensão estrutural, todas complementares. De facto, modelos bem estruturados permitem um bom desempenho dos cuidados de enfermagem que contribuem para maior independência e funcionalidade da família (Kholifah et al., 2016) mais capaz para gerir situações de transição normativas e não normativas, num ciclo entre estádios de entropia e neguentropia que permitem um crescente de complexidade evolutiva, tradutora da evolução do ciclo vital da família.

O MDAIF revelou-se eficaz na resposta às necessidades de saúde familiar, registando-se ganhos em saúde sensíveis aos cuidados de enfermagem, facto que é corroborado por Kholifah et al. (2016), Leahey \& Wright (2016), Shivalli et al. (2015) e Figueiredo (2012), que referem que os modelos de cuidados de enfermagem centrados na família são efetivos, oferecendo soluções viáveis e duradouras com envolvimento comunitário. A este propósito, Kokorelias et al. (2019) reforça a necessidade da existência de estudos que avaliem o impacte dos modelos nos resultados nos utentes, famílias ou sistemas de saúde dada a parca existência de estudos desta natureza.

$\mathrm{Na}$ dimensão estrutural registaram-se ganhos em saúde ao nível da segurança e zelo pelo edifício residencial, precauções de segurança, abastecimento de água e animal doméstico, traduzindo uma maior competência das famílias, nestas áreas. Estes 
resultados são corroborados por Russell et al. (2018), que referem que compreender a estrutura familiar de uma família ajuda os profissionais de saúde a responder de forma mais eficaz às necessidades de saúde das famílias contemporâneas.

Na dimensão desenvolvimental salienta-se a eficácia da aplicação do MDAIF na resposta às áreas de atenção: papel parental, satisfação conjugal e planeamento familiar. Segundo Hickey et al. (2018) os enfermeiros de família e de saúde pública possuem a habilidade de apoiar os pais durante a transição para a parentalidade, usando princípios e práticas informadas por evidências, ao mesmo tempo em que são sensíveis às necessidades exclusivas dos pais nas comunidades. Também Tavakol et al. (2017) salientam o papel dos terapeutas da família, na qual se inclui o enfermeiro de família, para o diagnóstico e intervenção com vista a maior satisfação conjugal e reduzir a taxa de divórcios.

Na dimensão funcional, registam-se ganhos em saúde no processo familiar (comunicação familiar, coping familiar, interação de papeis e relação dinâmica), resultados que são corroborados por Nourani, Seraj, Shakeri, \& Mokhber (2019) que referem que os profissionais de saúde podem ser agentes facilitadores da partilha de papeis na família com o objetivo de melhorar a satisfação conjugal. Shen et al. (2017) concluíram que após a intervenção dos profissionais de saúde baseada no modelo ecológico regista-se um maior tempo e qualidade de comunicação e uma melhor perceção da adequação da comunicação com impacte no bem-estar familiar. Por último, no papel de prestador de cuidados (conhecimento do papel, comportamento de adesão e saturação de papel) da dimensão funcional, verifica- se que os enfermeiros têm um papel efetivo na melhoria do bem-estar dos cuidadores, ajudando as famílias, dotando-as de mais conhecimentos para uma prestação de cuidados compartilhada e apoiando-as durante o processo de atendimento e segurança dos cuidados, tradutora de mais ganhos em saúde (Kuluski et al., 2019; Schaepe, Ewers, 2018; Imanigoghary, Peyrovi, \& Kazemi 2017). Regista-se como limitação ao estudo o reduzido número de famílias estudadas e o facto da amostra não ser representativa da população estudada.

\section{CONCLUSÃO}

O MDAIF enquanto modelo teórico e operativo constituiu a referência para que os estudantes de enfermagem avaliassem as famílias, identificassem áreas de atenção e formulassem diagnósticos de enfermagem, de forma colaborativa com a família, em contexto de Cuidados de Saúde Primários. Os estudantes a partir dos diagnósticos de enfermagem identificados selecionaram para cada diagnóstico as intervenções que se mostraram mais adequadas, de acordo com as preferências da família, de entre as sugeridas pelo MDAIF.

Dos diagnósticos de enfermagem formulados, antes e que acompanharam o após a intervenção, os que apresentaram maior magnitude foram os diagnósticos que integram a dimensão do desenvolvimento, seguidos dos da dimensão funcional e finalmente os da dimensão estrutural. Registaram-se ganhos em saúde sensíveis aos cuidados de enfermagem em todas as áreas de atenção das dimensões do MDAIF, com uma modificação positiva no estado dos diagnósticos de enfermagem de $80,8 \%$, do antes para após a intervenção. As famílias, com a ajuda dos 
estudantes de enfermagem fundamentados no MDAIF, souberam mobilizar as suas forças e os seus recursos que lhe permitiram dar resposta às suas necessidades, o que se traduziu em maior funcionalidade da família.

Os resultados podem informar o movimento em direção a um modelo de atendimento centrado na família, o MDAIF, pelo que se sugere a sua implementação disseminada na prática clínica junto dos enfermeiros de família e a realização de estudos de investigação, que sejam representativos da população, que sustentem a sua universalização.

\section{REFERÊNCIAS BIBLIOGRÁFICAS}

Corrêa, C. S., Queiroz, B. L. \& Fazito, D. (2016). Relação entre tamanho e estrutura da rede de apoio e o tempo individual dedicado à atenção ao idoso na cidade de São Paulo 2000. Revista Brasileira de Estudos de População, 33(1), 75-97. doi:10.20947/S0102309820160005

Estanque, E. (2017). Onde para a classe média? Breves notas sobre o conceito e a realidade portuguesa. Sociologia, Problemas e Práticas, 83, 37-54. doi:10.7458/SPP2017839971.

Figueiredo, M. H. (2012). Modelo Dinâmico de Avaliação e Intervenção Familiar: Uma abordagem colaborativa em enfermagem de família. Loures, Portugal: Lusociência.

Hickey, G., McGilloway, S., Leckey Y. \& Stokes, A. (2018). A universal early parenting education intervention in community-based primary care settings: Development and installation challenges. Education Sciences, 8(178). 1-15. doi:10.3390/educsci8040178

Imanigoghary, Z., Peyrovi, H. N. \& Kazemi, M. (2017). The role of nurses in coping process of family caregivers of vegetative patients: A qualitative study. International Journal Community Based Nursing and Midwifery, 5(1), 70-81. Recuperado de https://www.ncbi.nlm.nih.gov/pmc/articles/PMC521 9567/pdf/IJCBNM-5-70.pdf

Instituto Nacional de Estatística. Statistics Portugal. (2013). Famílias nos Censos 2011: Diversidade $e$ mudança. Destaque: Informação à Comunicação
Social, 1-26. Recuperado de https://www.ine.pt/xportal/xmain?xpid=INE\&xpgid=i ne_destaques\&DESTAQUESdest_boui=206614582\&D ESTAQUESmodo $=2 \&$ xlang $=p t$

Kholifah, S. N., Nursalam, M. N., Adriani, M., Ahsan, BN \& Susanto, T. (2016). Structural model for public health nurses' performance in the implementation of family nursing based on nursing relational capital. International Journal of Caring Sciences, 9(1), 914-26. Recuperado de http://www.internationaljournalofcaringsciences.org /docs/33_1-susanto_original_10_2.pdf

Kokorelias, K. M., Gignac, M. A, Naglie, G. \& Cameron, J. I. (2019). Towards a universal model of family centered care: a scoping review. BMC Health Services Research, 19(564), 1-11. doi: 10.1186/s12913-0194394-5

Kuluski, K., Kokorelias, K. M., Peckham, A., Goldhar, J., Petrie, J. \& Alloway, C. A. (2019). Twelve principles to support caregiver engagement in health care systems and health research. Patient Experience Journal, 6(1), 141-148. Recuperado de https://pxjournal.org/cgi/viewcontent.cgi?article=13 $38 \&$ context=journal

Leahey, M. \& Wright, L. M. (2016). Application of the Calgary Family Assessment and Intervention Models: Reflections on the reciprocity between the personal and the professional. Journal of Family Nursing, 22(4), 450-459. doi:10.1177/1074840716667972

Mauritti, R., Nunes, N. \& Alves, J. E. (2019). Social inequalities and development in Portugal: $\mathrm{A}$ look at the regional scale and the low density territories. Sociologia on line. Revista da Associação Portuguesa de Sociologia, (19), 102-126. doi: https://doi.org/10.30553/sociologiaonline.2019.19. 5

Nourani, Sh, Seraj F., Shakeri, M.T. \& Mokhber N. (2019). The relationship between gender-role beliefs, household labor division and marital satisfaction in couples. Journal of Holist Nursing and Midwifery, 29(1), 43-49. doi: 10.29252/HNMJ.29.1.301

Ordem dos Enfermeiros. (2007). Resumo mínimo de dados e core de indicadores de enfermagem para o repositório central de dados de saúde. Lisboa, Portugal: Autor. Recuperado em https://www.ordemenfermeiros.pt/arquivo/docume ntosoficiais/Documents/RMDE_IndicadoresVFOut2007.pdf 
Russell, L. T., Coleman, M. \& Ganong, L. (2018). Conceptualizing family structure in a social determinants of health framework. Journal of Family Theory \& Review, 10(4), 735-748. doi: 10.1111/jftr.12296

Schaepe, C., \& Ewers, M. (2018). "I see myself as part of the team" - family caregivers' contribution to safety in advanced home care. BMC Nursing, 17(40), 1-10. doi: 10.1186/s12912-018-0308-9

Seibel, B. L., Falceto, O. G., Hollist, C. S., Springer, P., Fernandes C. C. \& Koller, S. H. (2017). Rede de apoio social e funcionamento familiar: Estudo longitudinal sobre famílias em vulnerabilidade social. Pensando Famílias, 21(1), 120-136. Recuperado em https://lume.ufrgs.br/handle/10183/185176

Shen, C., Wan, A., Kwok, L.T., Pang S., Wang X., Stewart S.M., Lam TH \& Chan S.S. (2017). A community-based intervention program to enhance family communication and family well-being: The learning families project in Hong Kong. Front. Public Health, 5(257), 9. doi: 10.3389/fpubh.2017.00257
Shivalli, S., Majra,J. P., Akshaya, K. M. \& Qadiri, G. J. (2015). Family centered approach in primary health care: Experience from an urban area of Mangalore, India. The Scientific World Journal, 2015 (419192), 28. doi: $10.1155 / 2015 / 419192$

Silva, M. A., Costa, M. A., \& Silva, M. M. (2013). A Família em Cuidados de Saúde Primários: caracterização das atitudes dos enfermeiros. Revista de Enfermagem Referência, III Série (11), 19-28. doi: 10.12707/RIII13105

Tavakol, Z., Nasrabadi, A. N., Moghadam, Z. B., Salehiniya, H. \& Rezaei, E. (2017). A Review of the factors associated with marital satisfaction. Galen Medical Journal, 6(3), 197-207

Waldow, V. R. (2014). Cuidado colaborativo em instituições de saúde: A enfermeira como integradora. Texto Contexto Enfermagem, Florianópolis, 23(4), 1146-52. doi: 10.1590/0104-07072014001840013 